\title{
A Research on Anders-streben in The Secret History of the Mongols
}

\author{
Zhang Suojun ${ }^{1, a}$ \\ ${ }^{I}$ The Department of Foreign Languages, Hulunbuir University, Hailar, Inner Mongolia, China \\ Graduate University of Mongolia, Ulaanbaatar, Mongolia \\ ORCID ID: 0000-0003-4806-6407 \\ ageneralzhangsj@163.com
}

\begin{abstract}
With the help of the genre, the significance of the sign is more definite and the interpretation of the sign is much clearer. As a kind of indicative sign, the genre can guide the way to understand signs. If a sign text shows the pursuit of another genre, that is Anders-streben. The ancient Mongolian history work, The Secret History of the Mongols, belongs to a historical text in terms of the textual identity. Its characteristics should be objectivity, accuracy, conciseness, while the author pursues different genres. It is the pursuit of Anders-streben that improves the artistic quality, literariness and interestingness of its textual effects. Thus, it also promotes literary value, artistic value and research value of the great book. By deconstructing the efforts on Anders-streben made by the author, the research paper is to analyze novels, proses and legends and explore their special textual effects and even the theoretical mechanism behind it. Moreover, a new perspective is provided for appreciating and researching this historical book.
\end{abstract}

Keywords: Anders-streben, genre, textual effects, The Secret History of the Mongols

\section{《蒙古秘史》出位之思考辨}

张锁军 $^{1 \text {, a }}$

1 呼伦贝尔学院外国语学院, 海拉尔, 内蒙古自治区, 中国

蒙古国研究生大学, 乌兰巴托, 蒙古国

ORCID ID: 0000-0003-4806-6407

ageneralzhangsj@163.com

\section{摘要}

借助于体裁的符号表意更明确、符号意义的解释更清晰，作为指示性符号的体裁可以指引理解符号文本的路 径，而一个符号文本中所表现出来对另一体裁的追求即 “出位之思”。古代蒙古学巨著《蒙古秘史》其文本身 份属于历史文本，特点本应为客观性、准确性、简洁性等，作者却在记史的过程中追求体裁风格的转变，正是 作者对出位之思的追求增加了其文本效果的艺术性、文学性以及趣味性，从而提升了《蒙古秘史》不可估量的 文学价值、艺术价值以及研究价值。本文以小说体裁、散文体裁和神话体裁为例解构作者为出位之思所做的努 力, 以求挖掘其呈现的独特文本效果及其背后的理论机制, 为欣赏和研究这部史书提供新的视角。

关键词：出位之思，体裁，文本效果，蒙古秘史 


\section{1. 引言}

所谓体裁, 《现代汉语词典（第 7 版)》中这样描 述: “文学作品的表现形式。可以用各种标准来分了 如根据有韵无韵可分为韵文和散文; 根据结构可分为 诗歌、小说、散文、戏剧等” (2016: 1287), 可见, 这里的体裁是用来泛指一切文学作品的种类和样式。 体裁特征可以导致相同话语的不同理解, 英国学者昂 格尔 (Christoph Unger) 基于对关联理论的研究认为: 体裁在语言学领域、文学领域和翻译研究领域扮演了 重要角色（2006：1-3)。在符号学领域体裁应是 “符 号文本的文化类别” (赵毅衡 2011：124), 这里所说 的体裁范围更广，应包括诗歌、小说、电影、游戏、 音乐、绘画等所有的文化类别。符号表意与接收均可 以借助于体裁, 从符号学角度讲, 体裁既是指示符号, 更是最大规模的型文本, 规定了符号接收的最基本程 式, 或曰体裁决定了符号接收者解释符号文本的方式 或态度。同一个符号文本被写成诗歌的体裁或新闻报 道的体裁, 所引出的接收者阅读态度或理解期待必然 会有很大差别。同一符号文本中作者对不同体裁的追 求与努力这即是 “出位之思” (Anders-streben), 这个 词最早出现在德语中用于艺术批判的术语。符号文本, 特别是艺术文本, 总是希望有创意, 能够尽量摆脱各 种束缚, 出位之思势必成为其努力追求的目标之一。 然而, 事实上这里面有一个 “度” 的问题, 正所谓 “过 犹不及”, 如果一味地追求其他体裁而丧失了自我, 则得不偿失, 英国哲学家佩特 (Walter Pater) 早在 19 世纪下半叶就指出出位之思是 “部分摆脱自身局限” (1880：105)。可见, 巧妙地、得体地运用出位之思 才是符号文本的美好追求。

《蒙古秘史》也称作《元朝秘史》, 最初的版本是 由古蒙古文 (畏兀儿体) 所撰写完成, 现已失传, 此 后各类语言译本、各种形式版本众多。本文以巴雅尔 所编著的蒙文版《蒙古秘史》和阿尔达扎布《新译集 注<蒙古秘史 $>》$ 为底本, 对比前者蒙文版和后者译成 的现代汉语译文及注释, 考辨原著作者出位之思的追 求与应用, 即作者在记史中如何将小说、散文、神话 为代表的多种不同体裁巧妙结合在一起, 并以此来解 构《蒙古秘史》在记录蒙古民族生产生活、礼仪习俗、 战争谋略等诸多方面的同时, 其文本效果最终达成的 理论机制, 以求为更好地欣赏和研究这部民族历史巨 著做出贡献。

\section{2. 《蒙古秘史》历史体裁文本风格考究}

学界对于《蒙古秘史》的历史性和文学性都比较 认可, 但其文本身份到底是历史的还是文学的, 这个 问题 “国内学术界末有过强烈的争论, 不过纷纷表示 各自的立场, 选择自己的研究视角, 然后开展了进一 步的研究” (孛儿只斤・海燕 2019：158)。蒙古族古 代典籍 “文史不分家”, “这种传统从《蒙古秘史》发 端, 一直延续了数百年” (杭爱 1995: 24), 学者巴. 苏和也曾明确说道 “《蒙古秘史》是一部历史和文学
未分的蒙古族古代文献典籍”（2002：52)。笔者认为 仍有必要论证其文本身份或起主导作用的文本身份, 以明确读者的阅读期待。

无论《蒙古秘史》还是《元朝秘史》, 顾名思义, 其历史体裁的文本身份已然昭然若揭。“《蒙古秘史》 之所以称之为秘史，是官书，是黄金家族的秘密历史 或机密国史”（张锁军 2020：11），也即是说，其名字 俨然已经确定了其文本身份, 体裁的确定已经为读者 明确了读史的阅读态度。

历史文本的写作目的主要是为了记史, 客观性、 准确性、简洁性等均是历史文本的特点, 诚然, 这其 中依据不同的分类标准也可进行更细致地划分, 如: 正史和野史等, 但无论是哪一种历史文本, 其文本风 格是体裁早已规定好的, 这即是体裁的规定性。体裁 本身已经决定了文本的形式和风格，“同一段故事， 如果是历史书, 至少不能有太多的对话或场景描写, 也不能有太多的小人物命运，那是小说的形式特征”

（赵毅衡 2011：137）。这虽然算不上对历史体裁风格 的一个明确界定, 但也为历史体裁的文本风格确定了 大致方向。既然是以记史为主要目的, 那所记载的人 或事必定是历史上的较重要人物或较重大历史事件。

《蒙古秘史》全书共包括 282 节，从第 59 节至 268 节, 作者使用绝大部分篇幅来记录成吉思汗这个蒙古 族历史上重要人物的生平，从他降生、少年时代经受 折磨和历练、一统蒙古诸部落再到其扩大疆土的诸战 争, 直至最终逝世。所记载的其他历史人物, 要么是 蒙古黄金家族宗之谱系成员，要么是与历史特殊事件 相关联之成员, 其余人物要么省略, 要么一笔带过。 如: 都蛙.锁豁儿的记录, 虽出现次数不多, 却也同样 是为了缕清黄金家族宗亲关系。此外, 整部史书来看 对话及场景描写较多, 特别是针对某些历史事件, 这 与符号学家赵毅衡先生对历史文本的理解有悖, 如: 纳忽崖之战, 作者用对话叙述战争, 且运用大量修辞 文本来描述人物形象，但这些是为了借他人之口生动 叙述历史事件发生的过程及人物形象的塑造, 这也是 许多学者将其文本身份定位为历史文本和文学文本 二者兼具的原因之一，属于出位之思的体现。由此可 见, 若依据赵毅衡先生对历史体裁的认知为最基本的 要求, 这部史书大致符合历史体裁文本风格特征。

诚然, 若对《蒙古秘史》体裁最终界定, 还需要 更为完备的概念做指导。“史书体裁, 简称为史体, 是 指史书的外部表现形式。中国古代史籍浩如烟海, 史 书的体裁源远流长而多姿多彩, 最早有记言、记事两 种形式, 即所谓 “左史记言, 右史记事”, 后来随着历 史记载内容的不断丰富和史学的发展, 史学家们逐渐 创造出了编年体、纪传体、典制体、纪事本末体、史 评体、史考体、史注体、学案体，以及图表、谱牒、 笔记长编、纲目等多种形式” (白云 2018：65)。《蒙 古秘史》第二部分是全书的重点, 整体上看这部分是 成吉思汗的传记, 主要记述了成吉思汗及其身边主要 人员的事迹。该部分叙事以时间为轴，即编年体的文 本风格, 如: 鸡儿年(公元 1201 年)、狗儿年(公元 
1202 年)等, 但也可以说是以事件为叙事单元，即具 备纪事本末体的文本风格，如: 十三翼战争、阔亦田 之战等。综上分析可以看出其历史文本的特点显著, 考虑其文学价值不可忽略已然是事实, 笔者更倾向于 基于如下结论展开分析：《蒙古秘史》是一部文本身 份为历史体裁而兼具一定文学性和艺术性的历史著 作。

\section{3. 《蒙古秘史》出位之思及其文本效果}

《蒙古秘史》语言形式极其独特, 即使用通俗易 懂的“白话文”, 较客观地、生动地记录了当时蒙古社 会现状, 其体裁风格更是独具一格, 除了史学地位外,

《蒙古秘史》的文学性不容忽视, “《秘史》是公认的 蒙古文学经典作品, 书中有大量的韵文, 诗文并茂。 语言和形象具有草原民族特有的韵味。的确可以这样 说: 《秘史》对有些事件和人物的描述, 与其说是历史 的记录, 不如说是文学的创造” (亦邻真 1987: 77)。 接下来以极具代表性的小说、散文和神话体裁为例, 详细探讨《蒙古秘史》作者在体裁风格变换方面所做 出的具体努力及其所构建的文本效果, 即出位之思考 辨。

\section{1. 《蒙古秘史》之小说追求}

小说指 “一种运用文学语言的、叙事性的文学体 裁, 是以人物形象的刻画为中心, 通过一定的故事情 节和具体的环境描写, 形象而不是抽象地、生动而不 是枯燥地、深刻而不是肤浅地、既广阔而又多方面地 反映生活的文体” (赵建中 1990：450-451)。从定义 上来看, 小说体裁与散文相似, 但其特点是刻画人物 更生动、故事情节描写更逼真、场景环境描写更细致。 《蒙古秘史》中人物刻画最具典型性, 表现力强, 形 象突出, 人物对话有声有色, 对于史实情节和场景描 写也细致入微、淋漓尽致, 修辞手法运用居多, 等等 这些手法的运用, 都是作者对小说体裁的追求与努力。 这里仅试举一例, 可以彰显作者对小说体裁的追求。

《蒙古秘史》第 103 节, 䇝儿乞惕人为了报抢夺 诃额仑母亲的宿仇, 抓住了贴木真的妻子, 并将贴木 真的败军追至不儿罕-合勒敦山后, 才撤退离去。贴木 真从不儿罕山下来，说到：

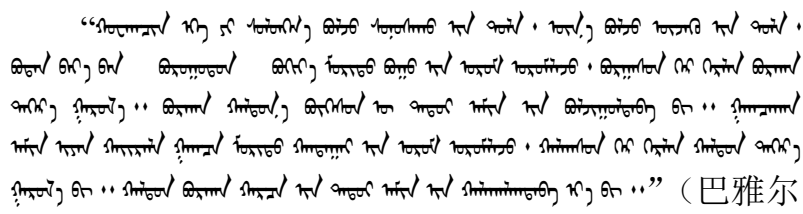

1981: 241-248)。

其现代汉语译文是 “由于豁阿黑臣老妇, 具有鼠 鼠般的听觉, 具有银鼠般的视觉, 才使我得以脱险! 骑着我仅有的驽马, 踩着鹿踏出的小径, 用柳条做住 室遮蔽, 不峏罕山保全了我, 我这小如闽子的性命! 爱惜我唯一的一条命, 骑着我仅有的一匹马, 寻着鹿 踏成的小径, 用杞柳编为茅舍, 登上了合勒敦山。合
勒敦-不峏罕山庇护了我, 我这小如燕禽的性命! ” (阿 尔达扎布 2005: 173-174)。

节选的这部分全部是由成吉思汗自言自语构成, 通过成吉思汗之口描述并刻画了豁阿黑臣老妇以及 自己脱险的整个过程, 语言简练, 描写细致。从修辞 格的使用上来看, 连用了四个修辞文本, 其中两个隐

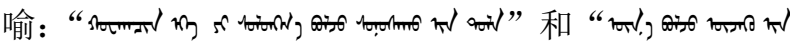
唍”, 现代汉语译文: “具有鼠鼠般的听觉”、“具有银

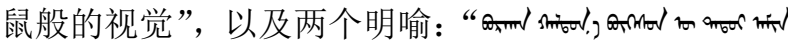

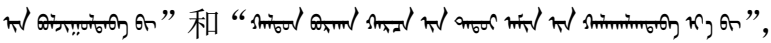
现代汉语译文: “我这小如虫子的性命”、“我这小如 燕禽的性命”。隐喻修辞格的使用使得豁阿黑臣老妇 的特点鲜明, 明喻修辞格的运用细致描写了当时成吉 思汗的心理状态, 总的来看, 修辞格运用有助于提升 人物形象的画面感。《蒙古秘史》中十分注重人物形 象的刻画，我国出版的《蒙古文学史》一书中，曾对 《蒙古秘史》中的人物形象做了专门论述, 可见其价

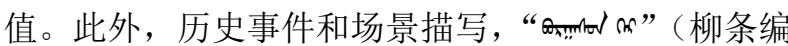

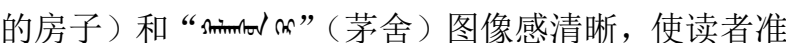
确捕捉到这些场景的特征，并通过人物形象刻画和场 景描写提升了历史事件叙述的细致程度, 细节描写更 加逼真。人物、事件和场景的描写即是作者对小说体 裁出位之思的追求, 这超越了普通历史文本的特征和 阅读期待, 使得文本效果形象、生动、细致、逼真, 文学性和艺术性的提升自不言而喻。

\section{2. 《蒙古秘史》之散文青睐}

散文是 “一种篇幅短小, 题材广泛, 写法自由, 文情并茂的文学体裁”（赵建中 1990：412），依据这 个定义来看, 散文体裁与小说体裁最大的差别是更注 重抒情。散文因篇幅短小故而语言凝练, 崇尚的是表 面 “无拘无束” 的写法，但实则 “乱中有序”，正所谓 “形散而神不散”，同时也侧重感情符号的投入。也 即是说, 无论记人还是记事都要有感情的投入，但是 感情不会无中生有, 要借助于合适的铺垫或场景的渲 染等。散文的语言特点以及叙事风格在《蒙古秘史》 文本中体现较为明显, 《蒙古秘史》既然是历史体裁, 那么记人和记事都是其必要组成部分。故此, 仅就某 一历史事件或历史事件中某一人物的叙述，其篇幅不 可能过长。至于其中作者 “写法自由”与 “文情并茂” 的风格, 笔者试举一例。

《蒙古秘史》第 145 节，成吉思汗负了伤，者勒 䉝赤身裸体迁入敌营为其寻找奶酪共饮（没找到马奶 子), 成吉思汗醒后对其大加赞赏，追诉其所立下的 三大功劳时，蒙古语原文这样说到:

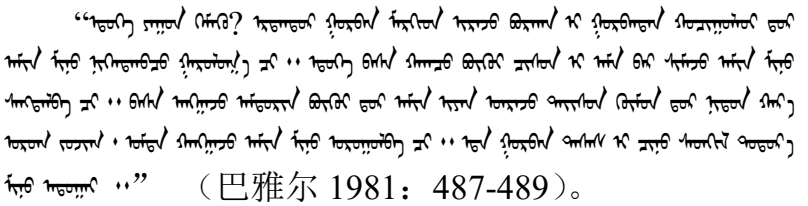


其现代汉语译文是 “现在我还需要说什么呢？以 前三部策儿乞惕前来[袭击我], 三次围搜不峏罕山的 时候，你曾救过我一次。如今又用嘴㕧吮淤血，使我 得以复苏。当我渴极了的时候, 你又不顾一切[舍命冒 险]跑进敌阵, 供我饮用, 使我渴解清醒。你这三次大 功, 将牢记在我的心中”（阿尔达扎布 2005: 266）。

这里不难看出, 成吉思汗对者勒䉝功劳的称赞与 肯定，成吉思汗历来对臣子是赏罚分明，当蒙古人建 立了自己庞大的帝国时, 成吉思汗歌颂了为此做出杰 出贡献的所有卫士和功臣。这里, 作者借成吉思汗之 口, 赞扬了者勒䉝的忠诚与勇敢, 通过成吉思汗感情 的抒发, 反应了蒙古民族心中的善和恶。“《秘史》作 者在人物矛盾冲突的某些关键时刻、事件进展的某些 重要场面, 作者或历史人物即打开情感的闸门, 或热 情赞颂, 或愤怒谴责, 或辛辣讽刺, 或坦诚明誓, 将 鲜明而奔放的感情尽情宣泄，使作品的节奏起伏跌宕， 事件生动逼真, 人物形象鲜明活泼” (张荣刚 2019: 63 )。这段叙述剪短自然, 充满感情, 称赞者勒策三大 功劳的句子内容与感情均层层递进, 呈现给读者一个 有血有肉的成吉思汗, 情感的注入赋予人物形象 “灵 魂”。更呈现了忠臣、良将与其之间的君臣之情, 感情 表达充沛, 皆因整个故事情节的前文铺垫, 即: 成吉 思汗颈部受伤, 危在旦夕。仅通过这一例, 不难看出 作者将抒情与叙事结合的写作手法, 这即是对散文体 裁的出位之思。散文文本的加入, 打破了读者原有阅 读历史文本的阅读期待, 其所实现的文本效果显著: 历史文本的叙述更具表现力和感染力, 文学趣味性更 明显, 艺术美感得到增强, 人物形象跃然纸上, 历史 事件生动逼真。

\section{3. 《蒙古秘史》之神话仰慕}

《蒙古秘史》文本内容上并不是简单的史实和人 物传记的记载, 其间不乏将历史事件和英雄传奇故事 集于一身, 这些文本内容的虽不是真实的历史, 但考 虑到古代蒙古民族崇尚天地的传统文化, 他们许多思 想和行为的来源正是依据神话、传说等, 这些神话文 本的加入符合当时人们思维习惯和心理特征, 或曰符 合作者真实记录蒙古人民生活的创作初衷。

《蒙古秘史》中记载的神话较多, 因篇幅所限, 下面仅就第 143 节颇具神话色彩地描述成吉思汗与 扎木合在阔亦田进行的大战为例, 论证作者的出位之 思，其蒙古文如下：

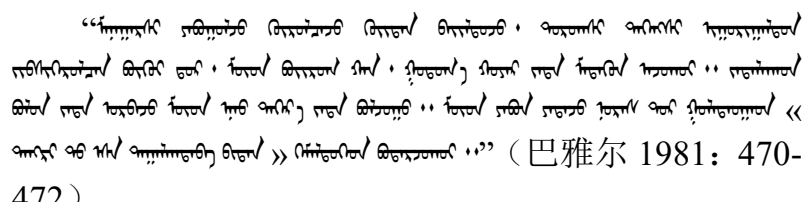

其汉语译文是: “第二天, 两军相会, 对阵在阔亦 田。在互相向上向下对峙, 双方拔剑张弓之际, 敌军 的不亦鲁黑和忽都合二人, 用札答石法术呼风唤雨。 施术的结果, 风雨却逆袭, 落到自己的头上。以致不
能逃脱，倒在沟㱡之中。他们便说: “我们没有被上天 喜欢”。于是, 便溃散而去了” (阿尔达扎布 2005: 259)。

短短一小段文本, 期间蕴含的符号信息却很丰富。 首先来看对于敌军不亦鲁黑和忽都合二人的描述, 二 人懂得呼风唤雨之术, 这是典型的神话文本体裁。此 外, 没想到风雨逆袭在自己军队身上, 导致失败, 他

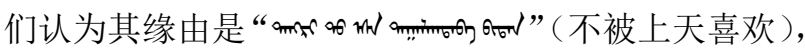
此句更是充满了对于上天、神灵的崇拜心理。神话、 传说是古代人们认知自然界以及生产、生活的重要方 式之一, 是人们用于解释许多自然现象以及社会文化 问题的元语言。“当一个社会需要 ‘神创” 或天命观来 建构其时空观和意识形态话语时, “神话” 是一种被 浸润在神圣或世俗表述中的不可或缺成分”（谭佳 2019: 51), 古时人们笃信神话、传说里的符号信息, 他们虽然未曾亲见神话记载的现象, 却更增加了对神 话符号文本神秘色彩的好奇与向往。《蒙古秘史》作 者在这里使用神话文本描述战争, 其所达成的文本效 果不仅是打破历史体裁所带来的阅读期待而提升了

《蒙古秘史》神话色彩, 使得阅读更具趣味性, 更多 的是通过对神话文本效果的追求来服务于作者的创 作主旨, 即: 围绕成吉思汗及其黄金家族记史。将神 话故事与历史史实交融在一起, 进一步突出了成吉思 汗人物的形象感、民族主义的认同感，同时也提升了 符号文本的文学价值和艺术价值。

《蒙古秘史》独特的体裁风格给人耳目一新的感觉, 让读者可以更生动的感受到许多历史人物活灵活现地 出现在眼前, 许多历史事件形象逼真地浮现在脑海, 增 加了读者阅读的兴趣。而对不同文学体裁的追求, 更进 一步提升了其文学价值和研究价值。联合国教科文组织 曾给予《蒙古秘史》高度评价, 认为其 “独特的艺术、 美学和文学传统及天才的语言, 使它不仅成为蒙古文学 中独一无二的著作, 而且也使它理所当然地进入世界经 典文学的宝库” (2001：32)。国际学界对《蒙古秘史》 各方面价值的肯定程度由此可见一斑。

\section{4. 结论}

任何符号文本的体裁对符号文本本身都具有约 束性和规定性, 出位之思仅是在其 “允许” 的范围内 巧妙为之, 以追求或努力实现不同的文本效果或意境。

《蒙古秘史》作为蒙古民族最主要的历史文献之一, 不仅较客观地记录了蒙古民族 500 多年的历史, 更在 记史过程中表现了对不同体裁的 “仰慕” 和追求, 这 正是其作者在不违背历史体裁要求的前提下, 对出位 之思的巧妙运用。无论这是作者有意为之还是无意所 为, 不争的事实是: 作者对出位之思的不断追求和努 力, 是提升其文本效果的艺术性、文学性以及趣味性 的根本原因, 从而构建了《蒙古秘史》不可估量的文 学价值、艺术价值以及研究价值。望本文可以从符号 学角度为《蒙古秘史》研究提供新的视野及有价值的 借鉴与参考, 为传播我国优秀的少数民族历史文化做 出相应贡献。 


\section{REFERENCES}

[1] Ardazhabu. (2005) New Translation to The Secret History of the Mongols with Intensive Notes. Inner Mongolia University Press. Hohhot.

[2] Ba, S.H. (2002) An Overview of Literature Study on The Secret History of the Mongols in $20^{\text {th }}$ Century in China. Studies of Ethnic Literature. 2:52.

[3] Bayar. (1981) The Secret History of the Mongols. Inner Mongolia University Press. Hohhot.

[4] Bai, Y. (2018) Chinese Historiography Thoughts. Historical Compilation Thoughts Volume. Fujian People's Publishing House. Fuzhou. 65.

[5] [5] Borzhijin, H.Y. (2019) Chinese Literature Studies on The Secret History of the Mongols within One Hundred Years (1917-2017). Studies of Ethnic Literature. 3:158.

[6] Hangai. (1995) On Documentary Literary Characteristics in The Secret History of the Mongols. Journal of Inner Mongolia Normal University (Philosophy and Social Science). 1:24.

[7] Evaluations on The Secret History of the Mongols from The United Nations Educational, Scientific and Cultural Organization. (2001) Inner Mongolia Social Sciences (Chinese). 4:32.

[8] Tang, J. (2019) The Study of Chinese Mythology for 70 Years. Folk Culture Forum. 51.

[9] Yilinzhen. (1897) The Secret History of the Mongols (Uighur Mongolian). Inner Mongolia University Press. Hohhot.

[10] Zhao, Y.H. (2011) Semiotics Principles and Problems. Nanjing University Press. Nanjing. pp. $124,137$.

[11] Zhao, J.Z. (1990) Textual Genre Studies. Nanjing University Press. Nanjing. pp. 412, 450-451.

[12] Zhang, S.J. (2020) Exploring Sign Lies in The Secret History of the Mongols. Journal of Hulunbuir University. 2: 11.

[13] Zhang, R.G. (2019) Style and Prose Features in The Secret History of the Mongols. Journal of Guiyang University (Social Science). 6: 63.

[14] Dictionary Editing Office, Institute of Linguistics, Chinese Academy of Social Sciences. (2016) Modern Chinese Dictionary ( $7^{\text {th }}$ Edition). The Commercial Press. Beijing. 1287.

[15] Walter Pater. (1980) The Renaissance Studies in Art and Poetry. Donald L. Hill. (eds). University of California Press. Berkeley and Los Angeles. 105.
[16] Christoph Unger. (2006) Genre, Relevance and Global Coherence: The Pragmatics of Discourse Type. Palgrave Macmillan. New York. pp.1-3. 